\title{
Erratum
}

\section{Is There a Potential Problem for the Potential Model?}

\author{
S. Chakrabarty
}

Z. Phys. C - Particles and Fields 28, 251 (1985)

In (1) and in $\alpha_{m}$ (after (6)), $\left(1+Q_{i} Q_{j}\right)$ should read $\left(1-\frac{3 \alpha}{4 \alpha_{s}} Q_{i} Q_{j}\right)$. The factor $\left(-\alpha_{m}\right)$ is missing from each of 5 th, 6 th and 7 th term on r.h.s. of (7). The r.h.s. of (8) should contain an extra factor of $\left(-1 /\left(\alpha_{m}\right)_{D^{0}}\right)$. In (9), 5th, 6th and 7th terms should be multiplied by $-\alpha_{m},-\alpha_{m}$ and $\alpha_{m} /\left(\alpha_{m}\right)_{D^{0}}$ respectively. In (10), (1 $\left.+3\left(M_{D^{*}}-M_{D}\right) / 4 m_{b}\right)^{-1}$ should be dropped and 5 th, 6th, 7th and 8th terms in the bracket should be multiplied by $-\alpha_{m}^{\prime},-\alpha_{m},-\alpha_{m}^{\prime}$ and $-\alpha_{m}$ respectively and extra term $3\left(M_{D^{*}}-M_{D}\right)\left(\alpha_{m}^{\prime} \frac{m_{c}}{m_{b}}-\alpha_{m}\right) / 4\left(\alpha_{m}\right)_{D^{0}}$ should be added to the r.h.s. On the r.h.s., the 4th and 5 th terms in (11), 1st terms in (12a) and (12b), 2nd terms in (13a) and (13b) should contain extra factors of $-\alpha_{m}, \alpha_{m} /\left(\alpha_{m}\right)_{D^{\mathrm{o}}}, \alpha_{m} /\left(\alpha_{m}\right)_{D^{\mathrm{o}}}, \alpha_{m}^{\prime} /\left(\alpha_{m}\right)_{D^{0}},-\alpha_{m}$ and $-\alpha_{m}^{\prime}$ respectively. In (14), $\left[1+m_{d}\left(M_{D^{*}}-M_{D}\right)\left(m_{b}\right.\right.$ $\left.\left.+m_{c}\right) / 8 m_{b}^{2} m_{c}\right]^{-1}$ should be dropped and $m_{c} m_{d}\left(M_{D^{*}}\right.$ $\left.-M_{D}\right)\left(\alpha_{m}^{\prime} / m_{b}^{2}-\alpha_{m} / m_{c}^{2}\right) / 8\left(\alpha_{m}\right)_{D^{\circ}}$ should be added to the r.h.s. In Sect. III, $a=5.4236 \times 10^{-3}$ should read $a$ $=1.036 \times 10^{-2}$. In (15) and (17), the numbers in the r.h.s. should read 3277.17 and 3229.19 respectively. The last relation should read

$\frac{1}{4 \cdot 16}\left(M_{K^{*}}-M_{K}\right)=M_{D^{*}}-M_{D}=4.47\left(M_{B^{*}}-M_{B}\right)$. 